\title{
Laboratory Observation of Coherent Structures beneath Microscale and Large-scale Breaking Waves under Wind Action
}

\section{Sang-Ho Oh}

Coastal Engineering Research Department, Korea Ocean Research \& Development Institute, Ansan P.O.Box 29, 425-600, Korea

Tel: 82-31-400-7802

Fax: 82-31-408-5823

E-mail: ohsangho@kordi.re.kr

\section{Kyung-Duck Suh}

Department of Civil and Environmental Engineering, Seoul National University, Seoul 151-742, Korea

Tel: 82-2-880-8760

Fax: 82-2-887-0349

E-mail:kdsuh@snu.ac.kr

\section{Natsuki Mizutani}

Department of Civil Engineering, Osaka Sangyo University, Nakagaito, Daito 574-8530, Japan

Tel: 072-875-3001

Fax: 072-875-5044

E-mail:mizutani@ce.osaka-sandai.ac.jp 


\begin{abstract}
This study presents the results of an experimental investigation of coherent structures generated beneath microscale and large-scale spilling breaking waves. By analyzing PIV velocity data of wind-generated waves that are acquired at three different wind speeds, the similarity and difference in spatio-temporal evolving characteristics of the coherent structures according to occurrence of the two types of wave breaking were investigated. There have been some researches on the coherent structures that are generated by microscale breaker. However, little has been known about the coherent structures formed underneath large-scale breaking waves. In this study, it was shown that a single strong coherent structure, whose direction of rotation of the coherent structure was the same as the wave orbital motion, is formed immediately underneath large-scale breaking crests. This was different from the processes occurring underneath microscale breakers that produce a series of coherent structures whose direction of rotation is not fixed. However, the overall evolving characteristics of the spatiotemporal evolution of the coherent structures were qualitatively the same between the two wave breaking events. As the breaker advances across the PIV field of view, the generated coherent structures interacted with pre-existing vortices and subsequently disappeared or reformed in the downwind of the breakers.
\end{abstract}

Keywords: coherent structures, particle image velocimetry, microscale wave breaking, large-scale wave breaking, vorticity 


\section{Nomenclature}

A

$d_{I}$

$d l$

F

$f_{d}$

$H_{S}$

$H_{r m s}$

M

$t$

$U$

$u$

$w$

$x$

z

$\Delta t$

$\Delta u$

Area surrounded by eight neighboring vectors $\left[\mathrm{m}^{2}\right]$

Size of a interrogation window [m]

Circumference surrounded by eight neighboring vectors [m]

Fetch length $[\mathrm{m}]$

Frequency of dominant wave component $[\mathrm{Hz}]$

Significant wave height [m]

Root mean squared wave height [m]

magnification factor [-]

Time $[\mathrm{s}]$

wind speed at $50 \mathrm{~cm}$ above the still water $[\mathrm{m} / \mathrm{s}]$

velocity in $x$ direction $[\mathrm{m} / \mathrm{s}]$

velocity in $z$ direction $[\mathrm{m} / \mathrm{s}]$

streamwise coordinate $[\mathrm{m}]$

vertical coordinate $[\mathrm{m}]$

time interval between light pulses [s]

velocity gradient $[1 / \mathrm{s}]$

Greeks
$\Gamma \quad$ Circulation $\left[\mathrm{m}^{2} / \mathrm{s}\right]$
$\lambda_{d} \quad$ Wavelength of dominant wave component $[\mathrm{Hz}]$
$\omega$
Vorticity [1/s]

$\left\langle|\omega|_{\max }\right\rangle \quad$ Ensemble average of absolute magnitude of the maximum vorticity [1/s] 


\section{Introduction}

When the wind continuously blows over a water surface, waves are generated and developed until they eventually break. Wave breaking plays a major role in limiting the growth of surface waves and enhancing air-water transfer of heat and gas through the generation of turbulent wake. In general, wave breaking occurs when some portion of the fluid near a wave crest spills or plunges down on the forward slope of the wave, frequently with entraining air into the water. Although this pattern of wave breaking is most obviously visible on the water surface, the occurrence of wave breaking without air entrainment is far more widespread, especially at low to moderate wind speeds.

A coherent structure is an organized large-scale deformable fluid parcel that is formed and long-lived in turbulent flow. It plays an essential role in large-scale dynamics of the turbulent flow and affects the motions of smaller-scale turbulence structures [1-2]. Coherent structures underneath wind-driven surface waves have been studied by several researchers [3-6]. Komori et al. [3] used a laser Doppler velocimeter to measure the water particle velocity below wind waves under mild wind conditions. They observed intermittent appearance of surface renewal rolling eddies in front of the wave crest and suggested that the eddies are generated by the strong shear due to the organized motion in the air flow. They also observed strong downward bursts that are presumed to be associated with the wave motion. Similar downward burst underneath the wind waves was investigated by Yoshikawa et al. [4] using high-sensitivity thermometer array in combination with a two-component sonic flowmeter. More recently, Siddiqui et al. [5, 6] visualized near-surface coherent structures underneath microscale breaking waves by simultaneous use of particle image velocimetry and 
infrared imagery. They found that the fractional area coverage of microscale breaking waves was closely correlated with the near-surface vorticity beneath the breaking waves. By correlating the magnitude of the near-surface vorticity with the air-water gas transfer velocity, they showed that microscale breaking waves are responsible for the enhancement of air-water gas transfer rates.

Most of these previous measurements were conducted at short fetch length $(F<10$ m) under relatively mild wind conditions $(U<10 \mathrm{~m} / \mathrm{s})$ where waves hardly or weakly break without visible whitecaps. This type of weakly breaking of short gravity waves with little air entrainment or spray is termed as microscale wave breaking, which is discriminated with large-scale wave breaking or whitecapping. Microscale wave breaking is characterized by a bore-like crest accompanied by parasitic capillary ripples distributed along the forward face $[7,8]$. The appearance of microscale wave breaking is much more frequent than large-scale wave breaking. In the laboratory, the frequency of occurrence of microscale breaking is already over $30 \%$ at wind speeds as low as $5 \mathrm{~m} / \mathrm{s}$ and reaches to $89 \%$ at wind speeds of $8.1 \mathrm{~m} / \mathrm{s}$ even at the short fetch of approximately 4 5 $\mathrm{m}[8,9]$. In contrast, the occurrence of large-scale wave breaking or whitecapping, which is commonly characterized by the white foam that is formed at the wave crest and spills over the front water surface, is rarely observed at the short fetch of the wind. In addition, whitecapping is likely to involve air entrainment into the water, which greatly disturbs the air-water interface and the internal flow field. Due to this extremity, it is difficult to measure near-surface flow field of large-scale breaking waves and much of their flow properties are yet fully investigated.

In this paper, the characteristics of coherent structures generated by the two different breaking phenomena, or microscale and large-scale wave breaking, were experimentally 
investigated. In a wind wave flume, waves were developed using moderate to high wind speeds $(U=7.5 \sim 14.0 \mathrm{~m} / \mathrm{s})$ and measured at a relatively long fetch length $(F=23.2 \mathrm{~m})$ to ensure the occurrence of microscale wave breaking for all the measured wave trains except a few wavelets of small amplitude while obtaining as many waves of large-scale breaking as possible. Using particle image velocimetry (PIV), the velocity fields in the streamwise vertical plane beneath wind waves were measured and near-surface coherent structures in the velocity fields were visualized by the vorticity-based conditional sampling [10]. Then, the differences in characteristics of the near-surface coherent structures according to the different wave breaking regimes were qualitatively investigated.

\section{Experiment}

The experiment was carried out in the wind-wave flume whose uniform test section is $1.5 \mathrm{~m}$ wide, $1.3 \mathrm{~m}$ high, and $28.5 \mathrm{~m}$ long as shown in Fig. 1. The sidewalls consist of glass plates and the top of the flume is covered with wood plates. Wind was generated by an axial fan driven by a $50 \mathrm{~kW}$ variable-speed motor at the upstream end of the flume. The wind then passes through guide vanes, a fine mesh screen, and honeycombs so that the wind velocity at the inlet section is quite uniform. At the inlet, a horizontal guide plate is installed, which can be adjusted vertically so as to be located at the water surface. The PIV measurement was carried out at two fetch lengths $(F=11.4 \mathrm{~m}$ and 23.2 m), where glass panes were installed at the bottom of the flume. In the present study, only the data obtained at the longer fetch were used in the analysis. The water depth was kept constant at $53 \mathrm{~cm}$, in which the spectral peak wave component always fell under 
the deepwater condition. The measurements were made with three different wind conditions. Wind was measured at 35-40 elevations within the range from the free surface up to $50 \mathrm{~cm}$ above the still water level, depending on experimental conditions. At each elevation, wind speeds were measured for $60 \mathrm{~s}$ at the sampling rate of $20 \mathrm{~Hz}$ to obtain the time-avergaed wind velocity. The experimental conditions and wave parameters are summarized in Table 1.

The schematic diagram of the experimental setup is shown in Fig. 2. A dark room was constructed in order to conduct the PIV measurement in daytime. In addition, a black thin plate was attached on the far sidewall of the flume to maximize illumination of seed particles. A 6W water-cooled argon-ion laser (Spectra-Physics Stabilite 2017) was used as a light source. The main body of the laser beam generator was located outside the darkroom and the laser light was carried to the desirable position by an optical cable. The laser beam was released vertically upward from underneath the glass pane and formed into laser sheet after passing a cylindrical lens and spherical optics. The laser sheet was projected along the centerline of the flume, parallel with the sidewalls. The thickness of the laser sheet was about $5 \mathrm{~mm}$ at still water surface. PIV images were captured using a charge-coupled device (CCD) camera (Photron FASTCAM-net) whose resolution is $512 \times 480$ pixels. The shutter speed was controlled by an electronic timing circuit. The maximum frame transfer rate of the PIV system was $250 \mathrm{fps}$ in full resolution. White polyvinyl chloride (PVC) particles (Shinetsu chemical co.) were used as seed materials. The mean diameter and the specific gravity of the particle in water were $150 \mu \mathrm{m}$ and 1.20 , respectively.

The CCD camera was positioned below the wave trough level with its angle of $20^{\circ}$ upward. In order to rectify the error in vertical scale due to photographing distortion and 
to compute the magnification factor of the PIV images, a grid paper was photographed while the wind fan was not in operation. After photographing the grid paper, wind was generated over quiescent water. To permit the slower traveling high-frequency component waves to travel to the measurement location, a sufficient waiting time was allowed to elapse after the initiation of wind generation. Seed particles were spread into water by hand about $20 \mathrm{~s}$ before recording PIV images and added repeatedly before each run. The field of view (FOV) of the camera was $23.3 \mathrm{~cm}$ wide and $21.8 \mathrm{~cm}$ high for all the test cases, and the corresponding image resolution was $0.455 \mathrm{~mm} / \mathrm{px}$. The size of FOV was determined so as to sufficiently capture waves under the severest experimental condition $(F=23.2 \mathrm{~m}$ and $U=14.0 \mathrm{~m} / \mathrm{s})$. The shutter speed and the frame transfer rate of the CCD camera were different depending on the experimental conditions as shown in Table 1 . The camera recorded PIV images at a constant frame transfer rate and shutter speed, and no frame straddling technique was used. A total of 2184 PIV images was captured continuously and stored in a built-in memory in real time and later downloaded to the hard disk of the host computer. The data acquisition was repeated eight times for $U=7.5 \mathrm{~m} / \mathrm{s}$ and 15 times for other two wind speeds. The total number of observed waves for each test case is shown in Table 1.

Together with the PIV system, a capacitance-type wave gauge was used to measure water surface displacement. The wave gauge was setup for its wire to be located at the center of the FOV longitudinally and $3 \mathrm{~mm}$ away from the edge of the laser sheet laterally as shown in Fig. 2. In this manner, synchronized image and wave data that had almost identical wave properties were obtained while the wire scarcely appeared in the captured images. The initiation of the PIV images and wave data acquisition was controlled by a trigger. Since the conventional wave gauge vibrates rapidly under wind 
action, the head amplifier of the wave gauge, the primary origin of the vibration, was separated from the main body and laid on the ceiling of the flume. The sampling rate of the wave gauge was $200 \mathrm{~Hz}$ for all the test cases.

\section{Data processing}

\subsection{Selection and classification of wave data}

The wave surface profile in a taken PIV image was identified by correlating the PIV image with the synchronously observed water surface displacement by the wave gauge. First, a rough estimate of the wave surface profile in the given PIV image was found by comparing the image with several precedent and next image frames. Since the wire of the synchronized wave gauge was positioned at the center of FOV as shown in Fig 2(b), the surface elevation at the center of the image must be equal to the surface elevation measured by the wave gauge at the recording instant. If we assume a wave travels without change of its shape during a short time interval, then it is possible to obtain the wave surface in the image using time history of the water surface displacement.

Near the free surface of water, the light sheet of laser was occasionally scattered owing to highly deformed water surface or more possibly foam particles on the surface. Then, extremely bright spots are seen in the PIV image frame caused by the reflection of laser light. In particular, when air bubbles generated at the tip of large-scale breaking crests were entrained into the water, the corresponding flow region in the PIV image frame was greatly contaminated over large spatial extent due to the strong reflection of laser light. Although such contamination of the PIV images mainly appears in the 
vicinity of large-scale breaking crests, it is also occasionally observed at either trough of large-scale breaking waves or crest of microscale breaking waves. Since velocity vectors at the contaminated area are likely erroneous, it is necessary to exclude the image frames if there are bright spots that may significantly affect the local velocity field. In this study, if the width or height of the contaminated area of the largest bright spot included in the water-side region of an image frame is greater than 32 pixels, the image frame was discarded. The threshold value of 32 pixels was chosen as the same length of the interrogation window, by which maximally four velocity vectors were absent or erroneously calculated owing to the bright spot contained in the PIV image. If three consecutive image frames that captured picture of a single wave were discarded, the whole image frames corresponding to the wave were excluded in the analysis.

As mentioned earlier, microscale wave breaking frequently occurs even for very young waves. In the laboratory, the percentage of occurrence of microscale wave breaking is already as high as $30 \%$ at low wind speeds $(U=4 \sim 5 \mathrm{~m} / \mathrm{s})$ and it reaches about $90 \%$ at moderate wind speeds $(U=7 \sim 10 \mathrm{~m} / \mathrm{s})$, even at very short fetch of wind $(F=4 \sim 5 \mathrm{~m} / \mathrm{s})[8,9]$. Hence, under present experimental condition $(U=7.5 \sim 14.0 \mathrm{~m} / \mathrm{s}$, $F=23.2 \mathrm{~m}$ ), almost all waves are expected to break either in microscale or large-scale pattern unless their amplitude is too small to break. Taking into account of this, some small wavelets whose height is lower than $1.5 \mathrm{~cm}$ were discarded in the analysis because they are less likely to break. Compared to earlier laboratory measurements of microscale wave breaking, the threshold value of $1.5 \mathrm{~cm}$ was sufficiently large to ensure the occurrence of microscale or large-scale wave breaking. In Table 2, the number of waves that were excluded due to both reasons mentioned above is shown, respectively.

Then, the remnant waves were classified into one of either microscale or large-scale 
breaking waves. The identification of large-scale breaking waves was made by inspecting appearance of apparent spilling foam or whitecaps immediately downwind of the wave crests in the PIV images as shown in Fig. 3. All the rest of waves were classified as microscale breaking waves. As shown in Table 2, the fraction of large-scale wave breaking increases with the wind speed. The probability of wave breaking indicated in Table 2 is generally consistent with previous laboratory experiments [1112].

3.2 Calculation of velocity field

The PIV images of the selected waves were analyzed by the image processing software, INSIGHT (TSI Inc.) after denoising the background noise of the raw images. The size of the interrogation window was $32 \times 32$ pixels and the window was overlapped by $50 \%$ with neighboring ones. This produces a $31 \times 29$ array of velocity vector maps for each PIV image, the resolution of which was $7.3 \mathrm{~mm} \times 7.3 \mathrm{~mm}$. The raw velocity vector matrix calculated by the software was further subjected to postprocessing in which spurious vectors owing to wrong evaluation of correlation-peak displacements were detected and replaced by alternative values.

The post-processing was done by using a MATLAB program written by the authors. First, erroneous velocity vectors whose magnitudes were greater than a limit value, which was found based on the global histogram plots as shown in Raffel et al. [13], were eliminated. The limit value was determined differently depending on the test cases. Second, a local median filter that is slightly modified from the filter of Westerweel [14] was used to remove velocity vectors that were not consistent in magnitude and direction 
with the neighboring velocity field in each $3 \times 3$ window. Special care was given to the velocity vectors right beneath the water surface. There were fewer than eight neighboring vectors, and the median filter might give skewed criteria, generally of smaller magnitude. To cope with this exceptionality, the topmost velocity vectors were compared with the extrapolated values based on the exponential fitting function based on the linear wave theory. If the magnitude of the topmost vector was less than 0.75 times the extrapolated value, the vector was replaced. Finally, all the removed spurious vectors were filled with new vectors calculated by weight-averaging the eight surrounding vectors of a $3 \times 3$ window. On average, the number of corrected spurious vectors was less than $3 \%$ of the total number of velocity vectors.

\subsection{Error analysis}

The uncertainty of the PIV measurement was investigated in terms of random errors and bias errors. Raffel et al. [13] showed the Monte-Carlo simulation results of the RMS random error with respect to the particle image diameter, density, and displacement. In the present PIV experiment, the diameter of particle image was approximately three pixels and the corresponding RMS uncertainty for $32 \times 32$ interrogation window was about 0.03 pixels. Similarly, the particle image density, 20 on the average for all the test cases, leads to the RMS error of 0.02 pixels. The RMS error due to the maximum particle image shift, which was about 10 near the wave crest, is 0.05 pixels. The combined error for these sources of error can be estimated by calculating the square root of the sum of the squared errors [15], and was estimated to be 0.062 pixels. This indicates that the expected total RMS error is no greater than $0.62 \%$ near the wave crest 
region.

On the other hand, the bias error due to the velocity gradient was estimated by applying a condition for the optimal PIV setup [16]. For a double-frame single-exposure system, the upper bound of the velocity gradient is estimated by the following inequality:

$$
|\Delta u|<\frac{0.03 d_{I}}{M \Delta t}
$$

where $d_{I}$ is the size of interrogation region in the pixel, $M$ is the magnification factor, and $\Delta t$ is the time interval between light pulses, or the inverse of frame transfer rate. The computed upper bounds of a velocity gradient, $|\Delta u|$, are $0.109,0.055$, and $0.026 \mathrm{~m} / \mathrm{s}$ for the frame transfer rate of 250,125 , and $60 \mathrm{fps}$, respectively. All the experimental data fall within these limits, so the bias error of the present PIV system can be considered negligible. Further details about the experimental data and post processing are described in Oh et al. [17].

\subsection{Detection of coherent structures}

The instantaneous vorticity normal to the plane of the light sheet was obtained at each grid point by calculating the circulation:

$$
\omega=\frac{\Gamma}{A}=\frac{1}{A} \oint u \cdot d l
$$


where $A$ and $d l$ respectively denote the area and circumference surrounded by the grid points of eight neighboring vectors. At the top points of a velocity map, right underneath the air-water interface where the number of the surrounding vectors is fewer than eight, the vorticity was calculated by assuming local continuity of the velocity field. Since the vorticity plot of the original spatial resolution (16 pixels) depicted rugged shape of vortices, the final vorticity field was calculated by the bilinear-interpolation of the vorticity data to a finer resolution by a factor of 16 (i.e. 1 pixel) as in Siddiqui et al. [5]. The vorticity map calculated by Eq. (2) displayed less-rugged shape of vortical structures in the map than the one calculated by the formulation of $\omega=\partial w / \partial x-\partial u / \partial z$, having almost the same magnitude of vorticity.

The coherent structures embedded in the flow field were detected using the vorticity-based conditional sampling method [10], which recognizes the coherent structures by applying a vorticity threshold and finding the points of higher vorticity than the threshold value. According to Bonnet et al. [18], commonly used detection techniques yield qualitative and quantitative agreement in detecting coherent structures. An exemplary snapshot of the coherent structures or vortices underneath large-scale wave breaking is shown in Fig. 4. Shown in the figure are the coherent structures whose absolute vorticity was greater than the background vorticity level [5], which was determined as the spatio-temporal mean of the absolute vorticity of the test case. Dashed-line contours denote clockwise vortices of negative vorticity whereas solid-line contours counter-clockwise vortices of positive vorticity. The white colored regions are the points where vorticity magnitude is lower than the background vorticity. 


\section{Results and discussion}

\subsection{Evolution of coherent structures}

Fig. 5 shows six instantaneous vorticity field during passage of one microscale breaking wave at the wind speed of $U=10.8 \mathrm{~m} / \mathrm{s}$. Also shown in the figure is the time history of water surface displacement where the observation time instances corresponding to the six vorticity snapshots are marked. The six snapshots of vorticity field are chosen so as to best illustrate spatio-temporal evolution of near-surface vortices during passage of the wave crest. Hence, the time intervals between the consecutive vorticity maps are not regular. Some of the near-surface vortices in Fig. 5 are labeled alphabetically in a capital letter to recognize them easily in the sequential plots. As shown in the figure, coherent structures were generated beneath the breaking wave crest and interaction of vortices actively occurred in the near-surface region during the wave passage, especially between the strong vortex beneath the wave crest and the vortices in front of it. As a result of the vortices interaction, the vortices downwind of the wave crest (vortices B, C, and D) were weakened or adjoined with neighboring vortices, and finally reformed behind the wave crest. Meanwhile, the coherent vortex A that was located at slightly deeper depth stayed at almost the same position without significant change of its shape during the advance of microscale breaker. This implies that the local vorticity at this depth was uncorrelated with dynamics of the wave that just passed the field of view (FOV).

The general feature of spatio-temporal evolution of coherent structures found in Fig. 5 is similar to the one reported in Siddiqui et al. [5,6]. They showed that persistent 
coherent structures are generated beneath the crest of microscale breaking waves and a wake is produced by a series of vortices that are formed behind the leading edge. Compared to their experimental results, however, the magnitude of vorticity shown in Fig. 5 is smaller while the size of vorticity is bigger. This is because the spatial resolution of our velocity data was approximately 3.5 times lower compared to their study. Hence, the vorticity magnitude of coherent structures was smaller since it is an averaged value over a larger spatial extent.

The spatio-temporal evolution of coherent structures beneath large-scale breaking waves at the wind speed of $U=14.0 \mathrm{~m} / \mathrm{s}$ is shown in Fig. 6. A large clockwise-rotating vortex labeled $G$ is formed immediately underneath the wave crest. As the wave crest advances across the FOV, the vortex G sequentially interacts with downstream vortices A, B, C, and D which were likely remnants of previous breaking waves. During the vortices interaction, co-rotating vortices $A$ and $B$ were adjoined with the vortex $G$ whereas counter-rotating vortices $\mathrm{C}$ and $\mathrm{D}$ were remained after passing the wave crest. Meanwhile, vortices $\mathrm{E}$ and $\mathrm{F}$ in the near bottom of the FOV slowly moved upwards during the passage of the breaking crest without significant vortices interaction around them.

The spatio-temporal evolution of coherent structures illustrated in Figs. 5 and 6 were typical features of microscale and large-scale wave breaking, respectively. By examining vorticity fields of all the analyzed waves, it was found that the evolution processes produced by both patterns of wave breaking are different in several respects. Beneath microscale breaking crest, several coherent structures were produced whose sizes were about $1 \mathrm{~cm}$ on average. The vorticity magnitude of the coherent structures was below $5 \mathrm{~s}^{-1}$ and dominant direction of rotation was not found. In contrast, when 
large-scale wave breaking occurs, one strong coherent vortex, approximately two to three times larger than coherent vortices of microscale breaking waves, was generated immediately underneath the wave crest. Most of the coherent vortex immediately underneath the wave crest rotated in clockwise direction and its vorticity typically ranged over $10 \mathrm{~s}^{-1}$. Another difference was the movement of coherent structures relatively far from the surface (e.g. vortices A in Fig. 5 and vortices $\mathrm{E}$ and $\mathrm{F}$ in Fig. 6). The coherent structures near bottom of the FOV were pulled up and gently interacted with neighboring vortices in compliance with the advance of large-scale breaking crest. This indicates that large-scale wave breaking influences to the vorticity field of the whole depth covered by the present FOV. In contrast, coherence structures that were not immediately underneath the surface stayed almost at the same location during advance of microscale breaking crest.

\subsection{Change in vertical vorticity profiles}

The evolution processes shown in Figs. 5 and 6 represent the Lagrangian change of coherent structures beneath microscale and large-scale breaking waves, respectively. On the other hand, the change of vorticity field in Eulerian viewpoint was investigated by plotting vertical vorticity profiles. In Figs. 7 and 8, the vertical profiles of the instantaneous vorticity during the passage of two patterns of breaking waves are shown at five locations across the FOV. The magnitude of the vorticity was expressed as the horizontal offset of each symbol from the dot point.

In Fig. 7, the vorticity profiles varied systematically according to the movement of surface wave in the near-surface region. In the six snapshots, it is clearly seen that the 
vorticity profiles became positively inclined with the occurrence of wave breaking and gradually returned to the similar profiles of the condition before entering of the breaking crest into the FOV (Fig. 6(a)). The change of the vorticity profiles was very significant approximately within the depth of the order of the significant wave height. In contrast, the vorticity profiles below this depth layer remained almost unchanged. At the location where vortices interaction occurred, a zigzag pattern of the vorticity profile was observed due to the presence of counter-rotating vortices around the region.

Meanwhile, the vorticity profiles underneath large-scale wave breaking significantly varied over the whole depth of the FOV as shown in Fig. 8. According to Toba and Yoshikawa [19], the depth layer of enhanced subsurface turbulence owing to the occurrence of wave breaking is five times the significant wave height, which is deeper than the depth covered by the present FOV. The vorticity profiles became negatively inclined with approaching of the breaking crest, showed a zigzag pattern, and returned to a neutral position after passage of the crest.

\subsection{Discussion}

When one of either microscale or large-scale wave breaking occurs, subsurface coherent structures were generated underneath and or slightly upwind of the breaking crests. During the both breaking processes, the coherent structures quickly lost its momentum and were left at the generated position, interacted with neighboring structures that were generated by previous breakers, and finally disappeared or reformed behind the breakers. As wave advances, successive new coherent structures were frequently generated at the leading edge of the breaker, which produced a wake of 
coherent structures that forms in line immediately underneath the free surface.

Although the origin of the coherent structures generated by microscale breaking waves has not been clearly understood, it is generally ascribed to the parasitic capillaries created and trapped on the forward slope of the crests that make the water mass behind them rotate via the surface tension effect [20]. Since the vortices are locally generated and quickly dissipated by viscosity, only the near-surface vorticity profiles within the depth of the order of the significant wave height are influenced as shown in Fig. 6. The rotation direction of the coherent structures generated by microscale breaking waves was examined for all the analyzed wave data. The result indicated that the rotation direction was alternating either counter-clockwise or clockwise depending on each individual breaker. This implies that the source of generating coherent structures (possibly the parasitic capillaries) acts on the water mass in opposite ways according to local wave dynamics.

The large-scale breakers observed in the present study were mostly spilling breakers. Air bubbles or droplets were frequently created on the wave surface and entrained into the water downwind of the wave crest. However, overturning jets falling on the free surface were scarcely observed. Compared to the microscale breakers, stronger and larger coherent structures occupied the whole FOV beneath the spilling breakers. In addition, the number and rotational direction of the coherent structures underneath the breaking crest were significantly different from those of microscale breaking waves. For approximately $88 \%$ of all the analyzed wave data, the spilling breakers produced only a single clockwise rotating coherent structure underneath its breaking crest. However, the overall evolving characteristics of the structure during the breaking processes were qualitatively similar to those of microscale breaking waves. This suggests that the basic 
nature of evolution of coherent structures after the generation would be not greatly different between the both breaking events.

The vorticity magnitude of the coherent structures presented in this study was much smaller, when compared to previous researches on microscale wave breaking [6,9]. As mentioned above, the main reason for this is comparatively low spatial resolution of the present PIV experimental setup. However, the resolution was sufficient for qualitatively examining general evolving processes of the coherent structures generated by both patterns of wave breaking. Another factor that may affect the magnitude of vorticity is the extrapolation of the velocity vector immediately underneath the water surface. As described in section 3.2, the extrapolation was made effect only when the magnitude of the topmost velocity vector is significantly less than the vectors below it. Since the extrapolation was based on the linear wave theory, the magnitude of the extrapolated vector and thus calculated vorticity might be smaller than the real value, particularly when large-scale wave breaking occurs.

As discussed in the above, the main interest in this study was to compare characteristics of the coherent structures generated by two patterns of breaking waves. Hence, the difference in the vorticity fields among the three experimental cases according to the wind speed was less investigated. One noteworthy thing regarding this is the rapid increase of the average of the maximum absolute vorticity, $\left\langle|\omega|_{\max }\right\rangle$, with the wind speed. As shown in Table 3, the value of $\left\langle|\omega|_{\max }>\right.$ increased by more than 2.5 times as the wind speed increased from 7.5 to $14.0 \mathrm{~m} / \mathrm{s}$. The increase rate is much higher than the rate of 0.4 presented in Siddiqui et al. [5], where the coherent structures were generated only by microscale breaking waves. 


\section{Conclusions}

The spatio-temporal evolving characteristics of the coherent structures generated by microscale and large-scale spilling wave breaking were investigated by performing the PIV measurement of the flow fields underneath both types of wave breaking. Previous experiments [5] have shown that coherent structures are formed behind the leading edge of the microscale breaker. Our measurements have shown that large-scale breaking waves also produce similar coherent structures immediately underneath their breaking crests. The overall characteristics of the spatio-temporal evolution of the coherent structures were qualitatively the same. In the wake of both types of breakers, the coherent structures interacted with neighboring vortices that were generated by previous breakers, and finally disappeared or reformed behind the breakers. According to the evolution of coherent structures, the near-surface vertical vorticity profiles changed systematically during the advance of breaking crests.

The number and rotation direction of the generated coherent structures were different between the two wave breaking events. Microscale breakers produce a series of coherent structures whose direction of rotation is not fixed as one of either counterclockwise or clockwise. In contrast, the spilling breakers mostly produce only a single clockwise rotating coherent vortex underneath its breaking crest. This suggests that the nature of generating coherent structures is different between the both breaking events. Owing to this difference, the vorticity profiles were negatively inclined with approaching of the spilling breaking crest. The change of vorticity profiles was significant approximately within the depth of the order of the significant wave height. Meanwhile, the vorticity profiles underneath large-scale wave breaking significantly 
varied over the whole depth of the FOV.

Acknowledgements

This research was supported by the Brain Korea 21 project. The first author was partly supported by the Port and Airport Research Institute (PARI) during his visit to Japan.

References

[1] A.K.M.F. Hussain, Coherent structures-reality and myth, Physics of Fluids 26 (10) (1983) 2816-2850.

[2] S.K. Robinson, Coherent motions in the turbulent boundary layer, Annual Review of Fluid Mechanics, 23 (1991) 601-639.

[3] S. Komori, R. Nagaosa, Y. Murakami, Turbulence structure and mass transfer across a sheared air-water interface in wind-driven turbulence, Journal of Fluid Mechanics, 249 (1993) 161-183.

[4] I. Yoshikawa, H. Kawamura, K. Okuda, Y. Toba, Turbulent structure in water under laboratory wind waves, Journal of the Oceanographical Society of Japan, 44 (1988) 143-156.

[5] M.H.K. Siddiqui, M.R. Loewen, C. Richardson, W.E. Asher, A.T. Jessup, Simultaneous particle image velocimetry and infrared imagery of microscale breaking waves, Physics in Fluids 13 (2001) 1891-1903.

[6] M.H.K. Siddiqui, M.R. Loewen, W.E. Asher, A.T. Jessup, Coherent structures beneath wind waves and their influence on air-water gas transfer, Journal of Geophysical Research, 109 (2004) C03024, doi:10.1029/2002JC001559.

[7] M.L. Banner, O.M. Phillips, On the incipient breaking of small scale waves, Journal 
of Fluid Mechanics, 65 (1974) 647-656.

[8] A.T. Jessup, C.J. Zappa, H. Yeh, Defining and quantifying microscale wave breaking with infrared imagery, Journal of Geophyisical Research, 102 (1997) 23145-23153.

[9] W.L. Peirson, M.L. Banner, Aqueous surface layer flows induced by microscale breaking wind waves, Journal of Fluid Mechanics, 479 (2003) 1-38.

[10] A.K.M.F. Hussain, M. Hayakawa, Eduction of large-scale organized structures in a turbulent plane wake, Journal of Fluid Mechanics, 180 (1987) 193-229.

[11] P.A. Hwang, D. Xu, J. Wu, Breaking of wind-generated waves: measurements and characteristics, Journal of Fluid Mechanics, 202 (1989) 177-200.

[12] D. Xu, P.A. Hwang, J. Wu, Breaking of wind-generated waves. Journal of Physical Oceanography, 16 (1986) 2172-2178.

[13] M. Raffel, C.E. Willert, J. Kompenhans, Particle Image Velocimetry, Spinger, Berlin, 1998.

[14] J. Westerweel, Efficient detection of spurious vectors in particle image velocimetry data, Experiments in Fluids, 16 (1994) 236-247.

[15] P. Dong, T.-Y. Hsu, P. Atsavapranee, T. Wei, Digital particle image accelerometry, Experiments in Fluids, 30 (2001) 626-632.

[16] R.D. Keane, R.D. Adrian, Theory of cross-correlation analysis of PIV images, in: F.T.M. Nieuwstadt (Ed.), Flow Visualization and Image Analysis, Kluwer, Dordrecht, 1993, pp.1-25.

[17] S.-H. Oh, N. Mizutani, K.-D. Suh, N. Hashimoto, Experimental investigation of breaking criteria of deepwater wind waves under strong wind action. Applied Ocean Research, 27 (2005) 235-250.

[18] J.P. Bonnet et al., Collaborative testing of eddy structure identification methods in 
free turbulent shear flows, Experiments in Fluids, 25 (1998) 197-225.

[19] Y. Toba, H. Kawamura, Wind-wave coupled downward-bursting boundary layer (DBBL) beneath the sea surface, Journal of Oceanography, 52 (1996) 409-419.

[20] M.S. Longuet-Higgins, Capillary rollers and bores, Journal of Fluid Mechanics, 240 (1992) 659-679. 
Table 1. Experimental conditions and wave parameters

\begin{tabular}{lccc}
\hline & \multicolumn{2}{c}{ Experimental conditions } & \\
$U(\mathrm{~m} / \mathrm{s})$ & 7.5 & 10.8 & 14.0 \\
Frame rate $(\mathrm{fps})$ & 60 & 125 & 250 \\
Shutter speed $\left(10^{-3} \mathrm{~s}\right)$ & 4 & 4 & 2 \\
Number of runs & 8 & 15 & 15 \\
& & & \\
$H_{s}(\mathrm{~cm})$ & Wave parameters & & \\
$H_{r m s}(\mathrm{~cm})$ & 3.00 & 5.22 & 7.84 \\
$f_{d}(\mathrm{~Hz})$ & 2.06 & 3.81 & 5.87 \\
$\lambda_{d}(\mathrm{~m})$ & 2.57 & 2.01 & 1.82 \\
\hline
\end{tabular}


Table 2. Number of the observed, removed, and analyzed waves

\begin{tabular}{lccc}
\hline$U(\mathrm{~m} / \mathrm{s})$ & 7.5 & 10.8 & 14.0 \\
\hline Observed waves & 739 & 480 & 208 \\
Removed waves & 240 & 89 & 59 \\
- due to reflection of laser light & $(4)$ & $(46)$ & $(48)$ \\
- due to smaller wave height than threshold & $(236)$ & $(43)$ & $(11)$ \\
Analyzed waves & 499 & 391 & 149 \\
- microscale breaking & $(490)$ & $(355)$ & $(116)$ \\
- large-scale breaking & $(9)$ & $(36)$ & $(33)$ \\
\hline
\end{tabular}


Table 3. Wave parameters of the analyzed waves

\begin{tabular}{lccc}
\hline$U(\mathrm{~m} / \mathrm{s})$ & 7.5 & 10.8 & 14.0 \\
\hline$H_{r m s}(\mathrm{~cm})$ & 2.41 & 3.98 & 6.05 \\
$f_{d}(\mathrm{~Hz})$ & 2.39 & 1.91 & 1.76 \\
$\lambda_{d}(\mathrm{~m})$ & 0.27 & 0.43 & 0.51 \\
$<|\omega|_{\max }>$ & 4.1 & 6.6 & 10.7 \\
\hline
\end{tabular}




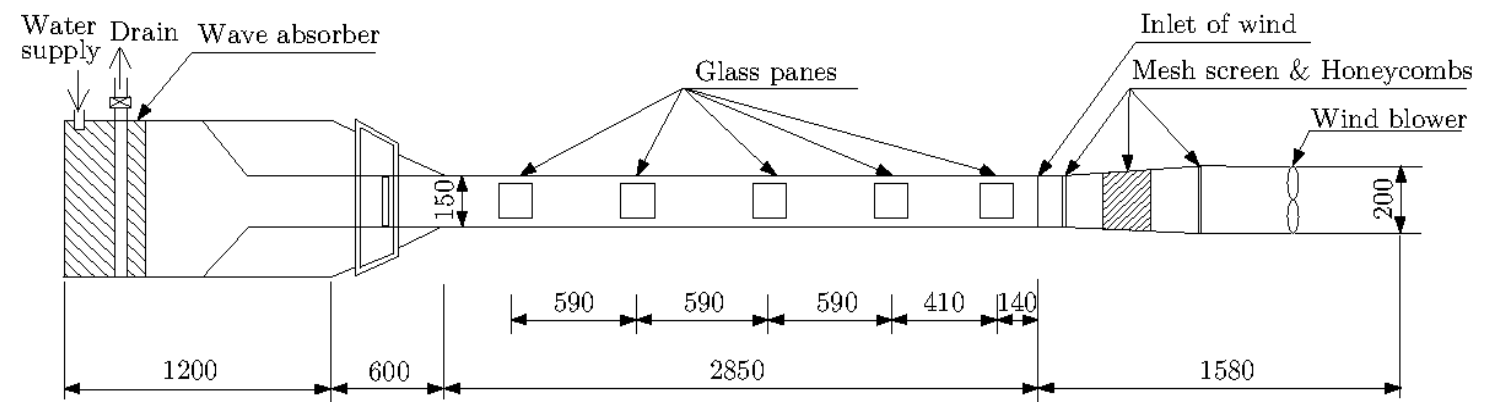

Fig. 1. Plan view of the wind-wave flume 

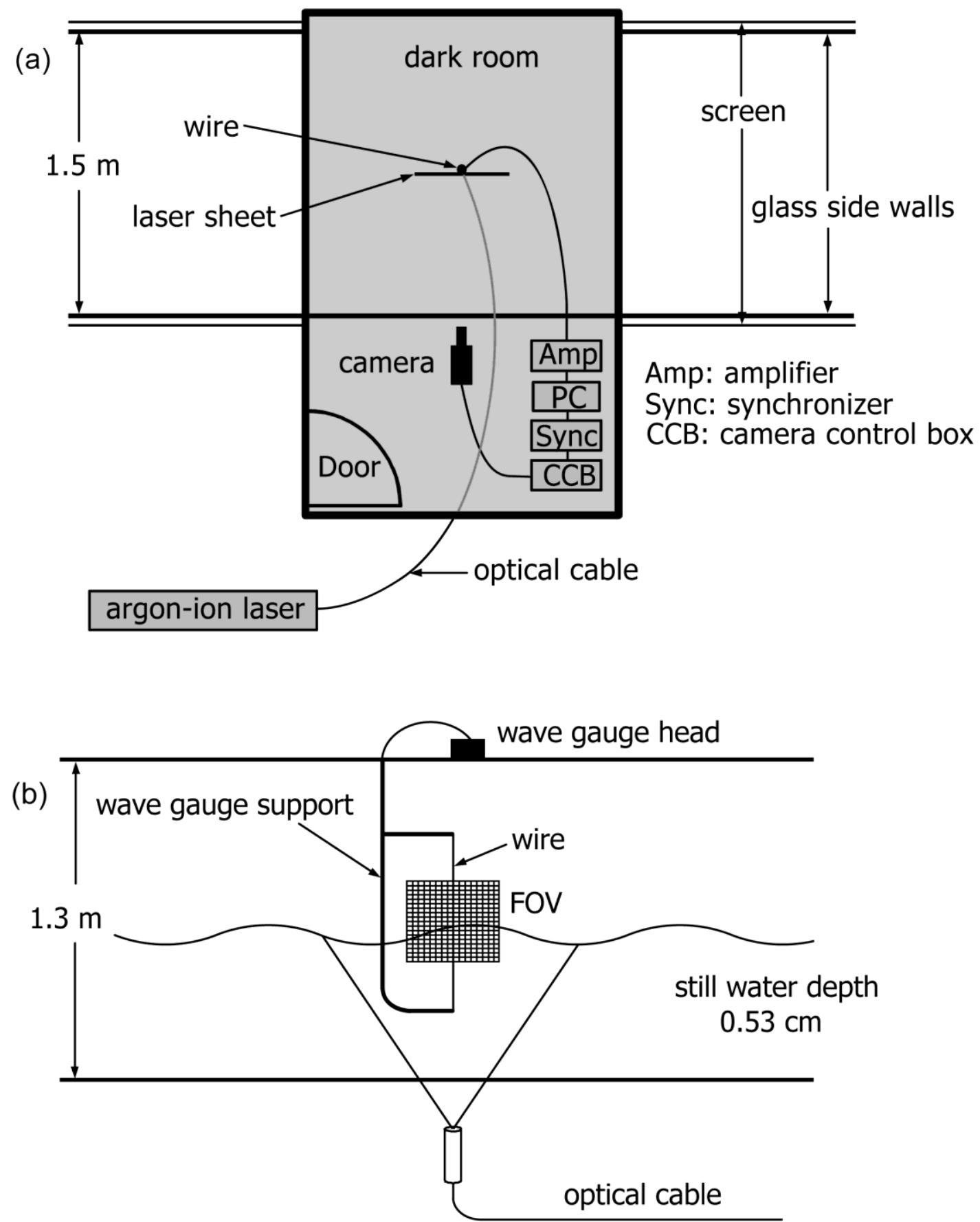

Fig. 2. Experimental setup. (a) Plan view, (b) Side view. 


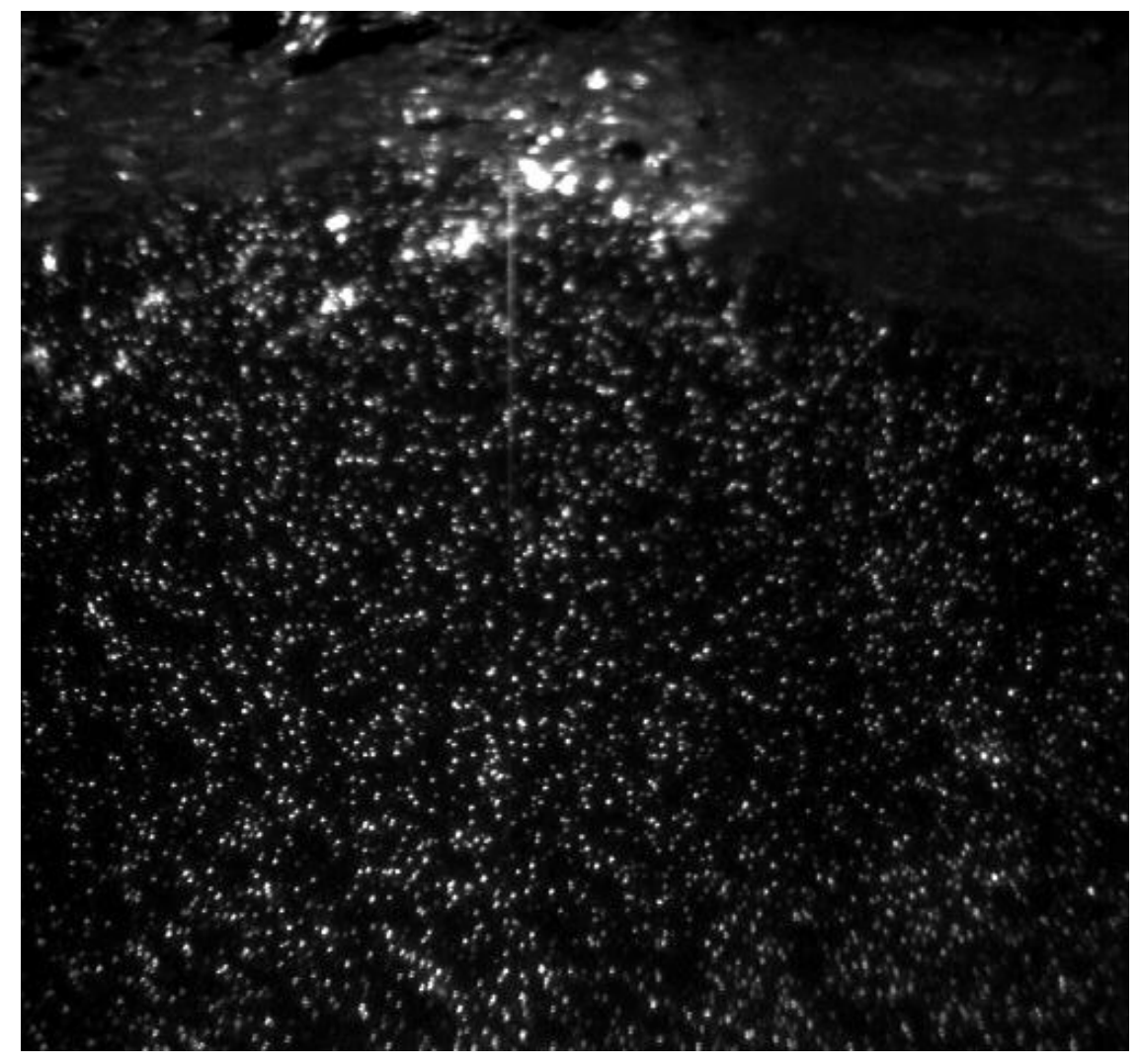

Fig. 3. An exemplary PIV image showing whitecaps near the wave crest. 


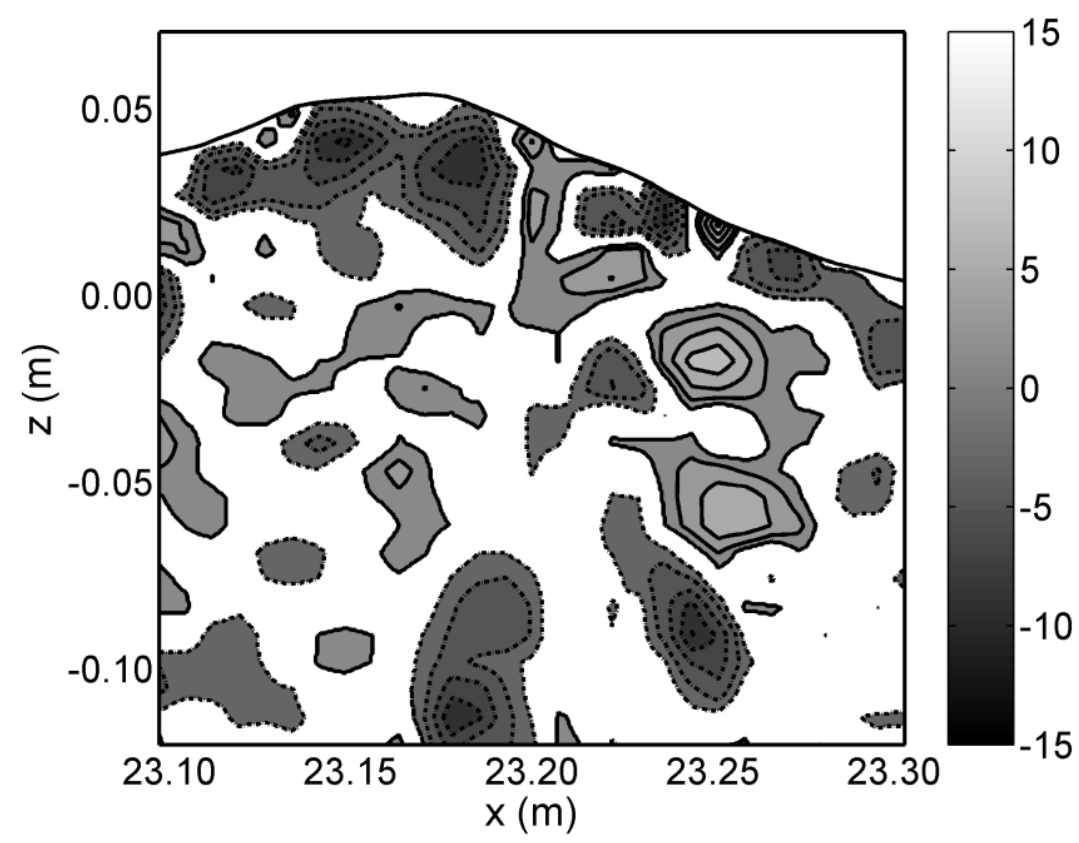

Fig. 4. An example of the instantaneous vorticity field. 

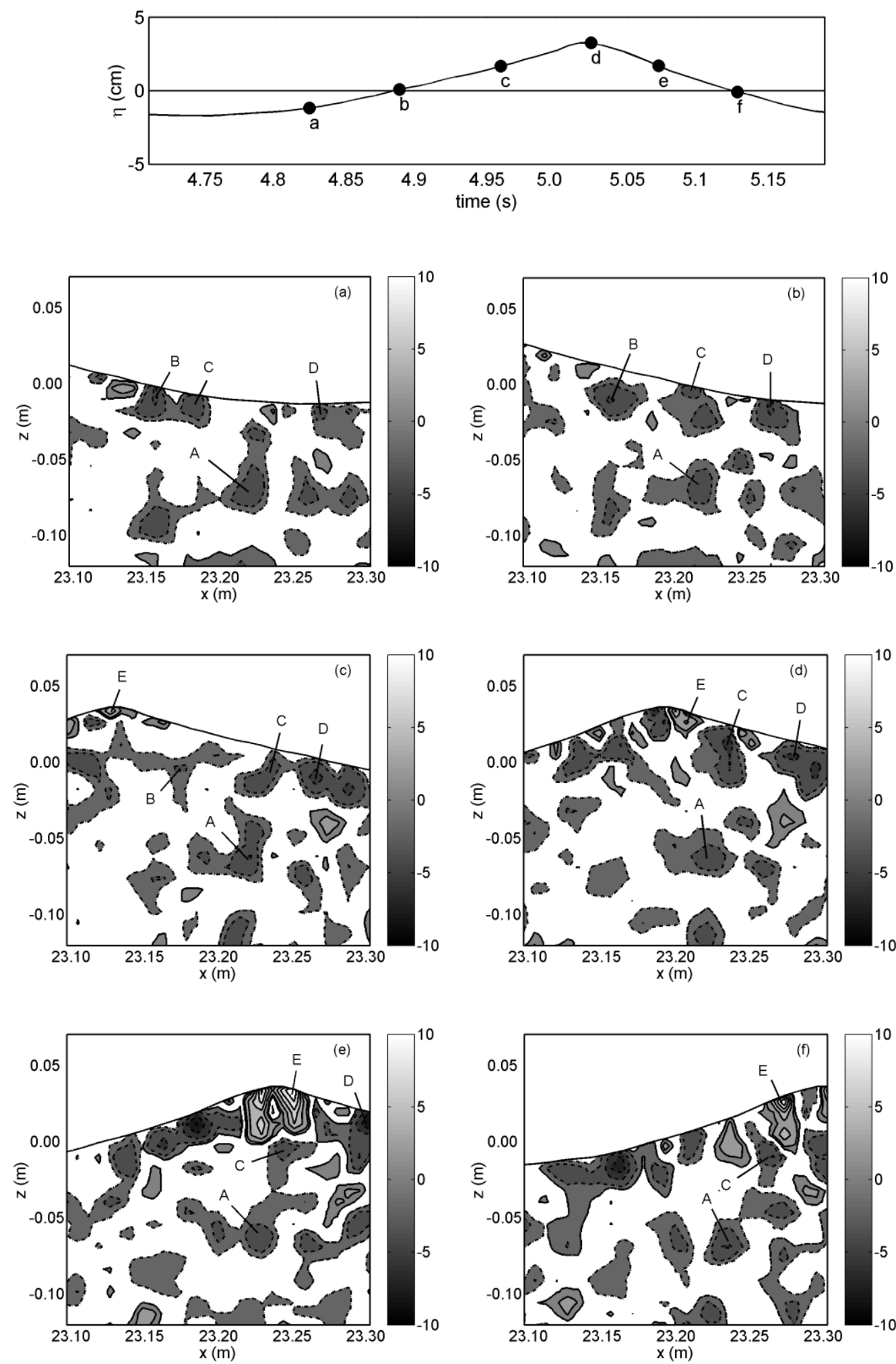

Fig. 5. Spatio-temporal evolution of coherent structures generated by microscale breaking wave. 

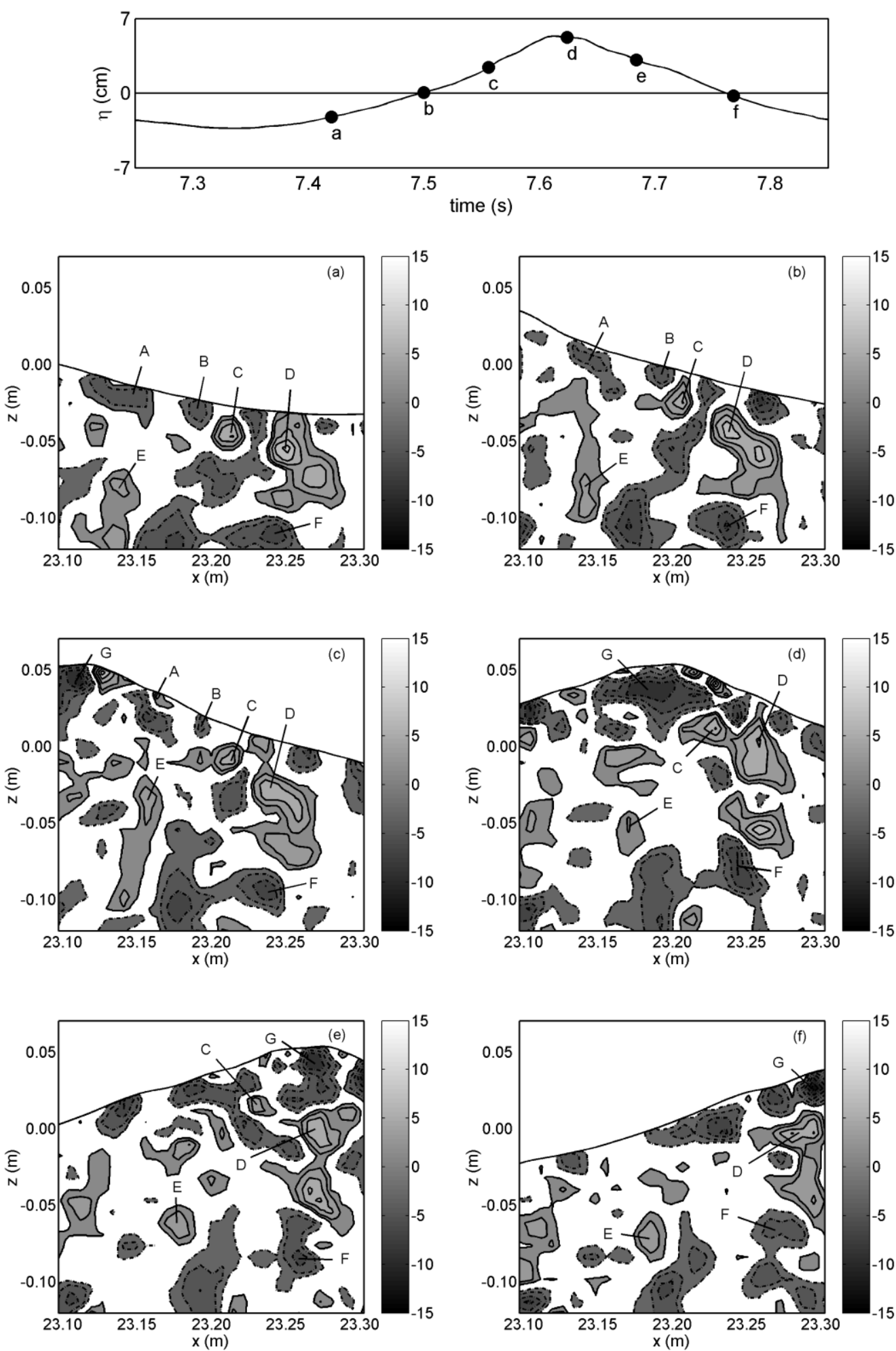

Fig. 6. Spatio-temporal evolution of coherent structures generated by large-scale breaking wave. 

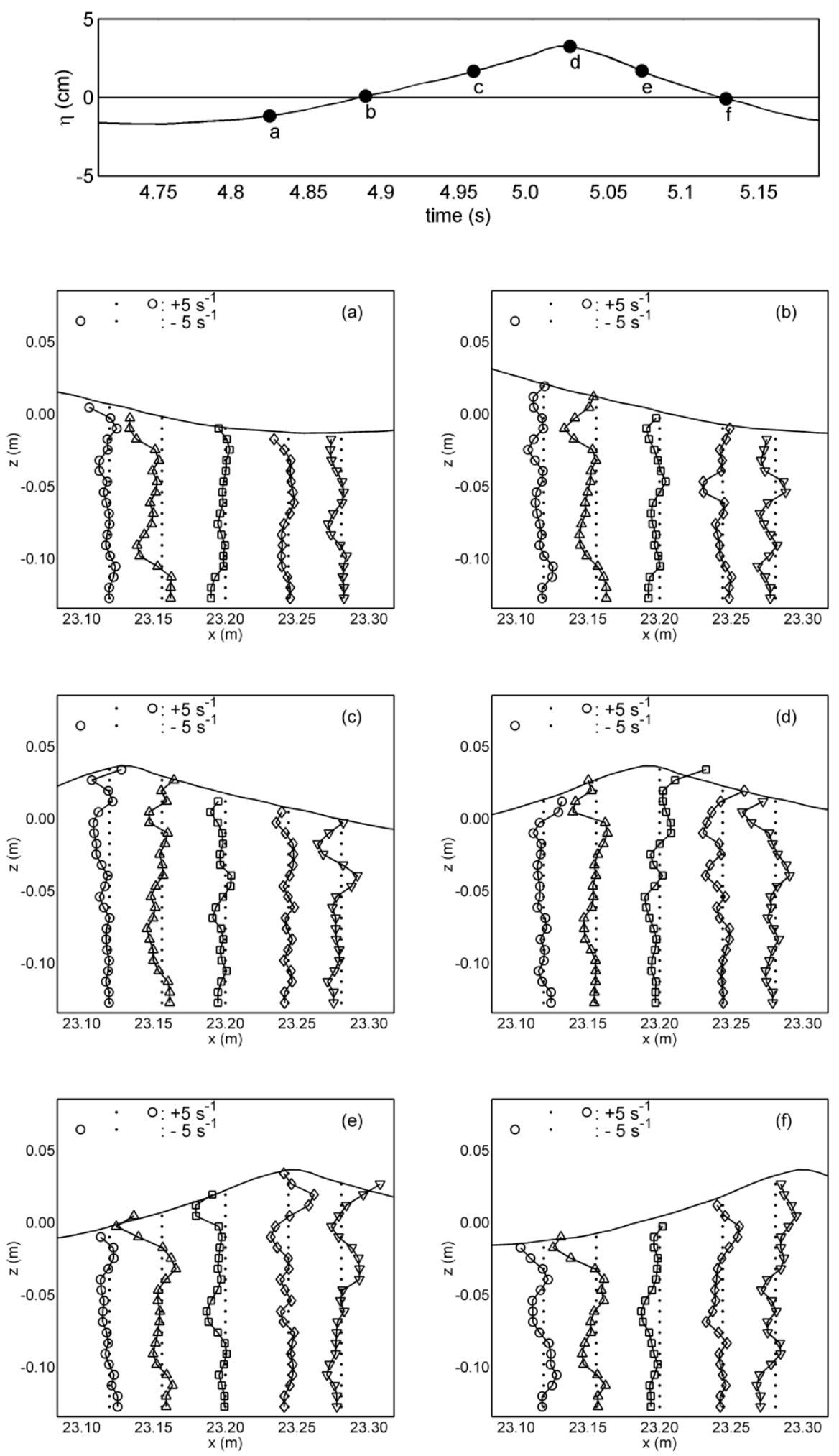

Fig. 7. Change of vertical vorticity profiles underneath microscale breaking wave. 

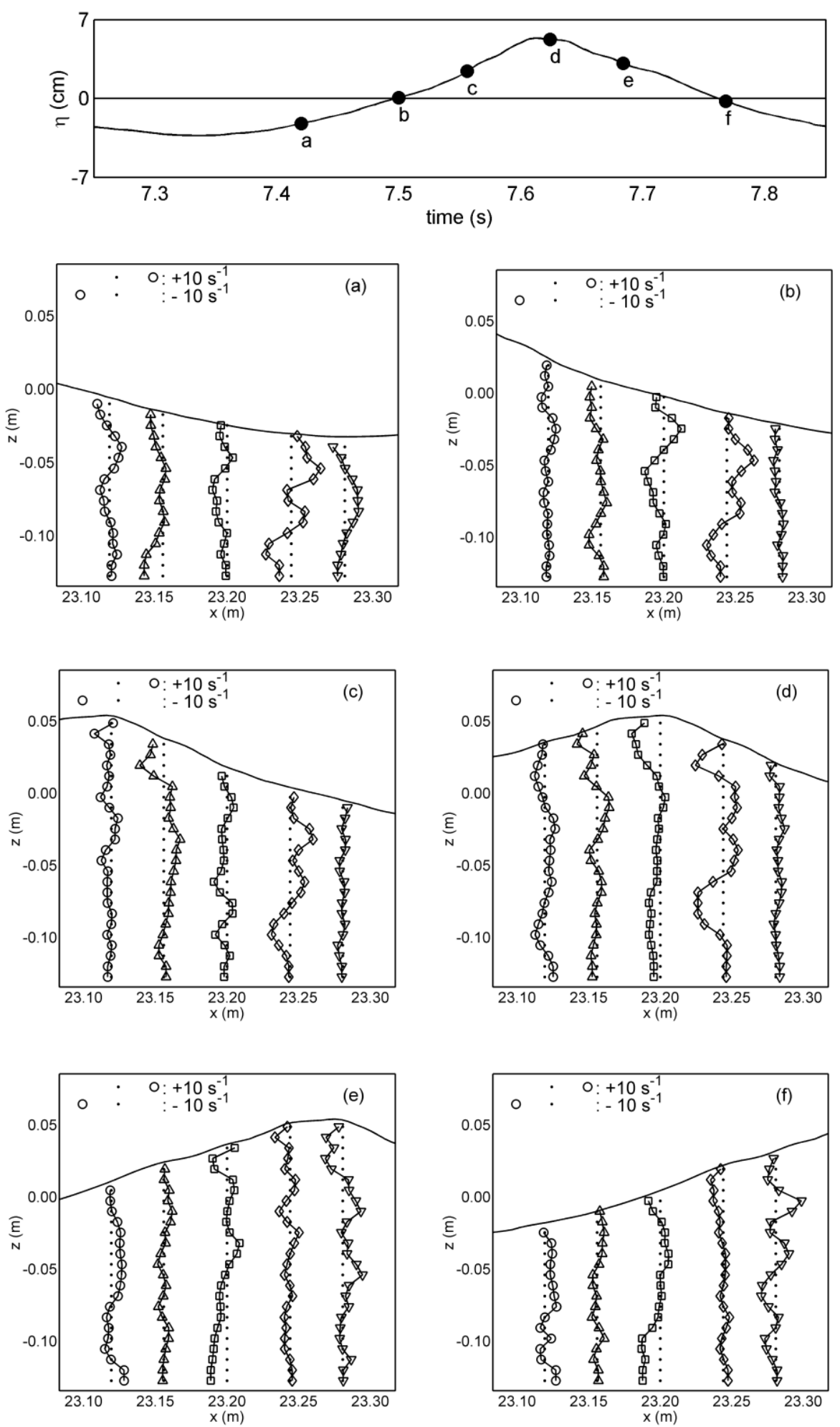

Fig. 8. Change of vertical vorticity profiles underneath large-scale breaking wave. 